\title{
STUDENT ROLE ADJUSTMENT IN ONLINE COMMUNITIES OF INQUIRY: MODEL AND INSTRUMENT VALIDATION
}

\author{
D. Randy Garrison \\ University of Calgary \\ Email: garrison@ucalgary.ca
}

Martha Cleveland-Innes

Assistant Professor

Athabasca University

Dr. Tak Fung

Senior Statistical Consultant, Information Technologies

University of Calgary

\begin{abstract}
The purpose of this study is to validate an instrument to study role adjustment of students new to an online community of inquiry. The community of inquiry conceptual model for online learning was used to shape this research and identify the core elements and conditions associated with role adjustment to online learning (Garrison, Anderson and Archer, 2000). Through a factor analytic process it is shown that the instrument did reflect the theoretical model. It was also useful in refining the items for the questionnaire. The instrument is for use in future research designed to measure and understand student role adjustment in online learning.
\end{abstract}

\section{KEYWORDS}

Role adjustment, community of inquiry, learning effectiveness, online learning

\section{INTRODUCTION}

This is the first phase of an exploratory study to understand and explain the conditions under which students adopt the role identity of online learners. By "online learner" we mean a student who participates in an asynchronous educational environment that capitalizes on Internet communication technologies to access information and create an engaged community of inquiry and discourse. Like any social process, role identity adjustment is dependent upon social forces that will allow social identity to emerge. Critical to the development of social role identity is engagement in a community, in this case, an online community of inquiry. The focus of the broader study is to explore the factor structure of the hypothesized online community of inquiry and its ability to assess role identity adjustment.

In the move to a collaborative online educational experience, role adjustment is inevitable. This is based first upon the reality that online learning is facilitated largely in an asynchronous text-based environment. Online learning necessitates a qualitative shift in the nature of the communication and interaction. There 
is a fundamental change in the control of time and the nature of the multi-dimensional (voice, word, image) interaction [1]. Secondly, for an online learning experience to reach its educational potential, a learning community must be established and sustained. In this regard, online learning represents a new "learning ecology" [2] where students interact in a reflective manner.

Asynchronous collaborative learning is a flexible and open system that necessitates rethinking the role of student. This interactive complexity represents a different kind of cognitive, social and teaching presence and brings with it the need for appropriate role adjustment. Students are required to learn new protocols and expectations. The roles in an online educational community of inquiry necessitate considerable adjustment from those of spontaneous, verbal face-to-face conversations. Understanding the intricacy of this adjustment is an important element in designing and delivering meaningful learning experiences online.

The purpose of the research reported here is to explore the factor structure of an instrument constructed to assess role adjustment in an online asynchronous community of inquiry. For the purposes of this particular study, the question is whether the instrument reflects the hypothesized constituent elements of the conceptual model. Through the factor structure and item analysis the goal is to produce a valid instrument, which would be used to measure role identity adjustment for learners as they adjust to an online learning environment. The instrument was constructed from the community of inquiry model developed by Garrison, Anderson and Archer [3]. This model comprehensively represents the online learning environment.

\section{THEORETICAL FRAMEWORK}

Two theoretical perspectives inform this research. The first associated with online communities of inquiry sets the stage from an educational perspective. The second perspective builds upon the work of Collier [4] with regard to student role adjustment. Together, they provide a new perspective on online learning in terms of how students cope with adjusting to what is a very different educational approach and transaction. This framework also provides a rich new insight with which to interpret and understand the findings of this study.

\section{A. Community of Inquiry}

Online educational communities have the properties of being both reflective and interactive. That is, individuals have the freedom of private reflective thought equitably balance with interaction in the public sphere. This is made possible through the written word and communication networks. Arguably, this reliance upon collaborative written communication lends itself to concurrent critical reflection and discourse - and ultimately to higher-order learning outcomes.

The model used here to represent this complex online community of inquiry is represented in Figure 1 [5]. There are three core elements to this online community-cognitive, social and teaching presence. The triadic structure of the model emerged from the educational literature and the experience of its authors. Together, it is hypothesized that they represent the primary dimensions of role adjustment in an online educational community. Moreover, an online community of inquiry will inevitably require adjustments in terms of cognitive, social and teaching presence. 


\section{Community of Inquiry}

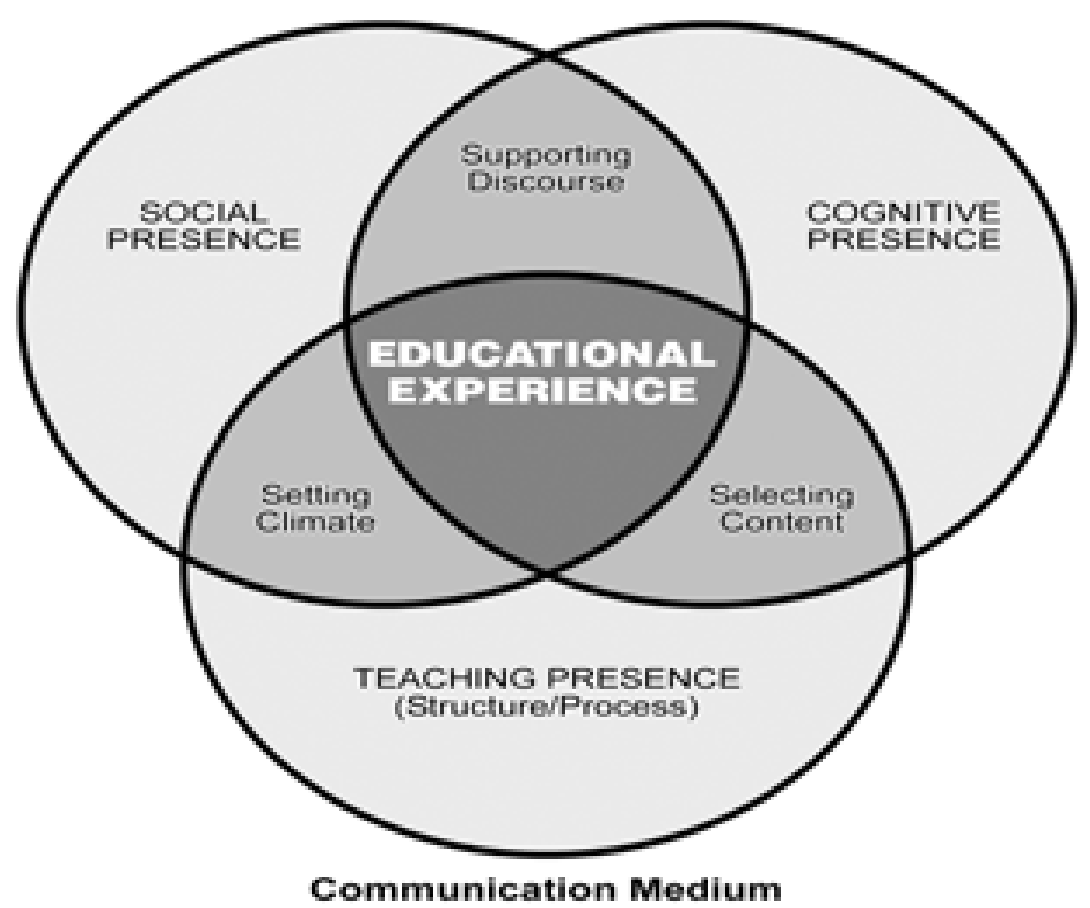

Figure 1: Community of Inquiry

(Reproduced by permission from Pergamon.

From Garrison, D. R., Anderson, T., \& Archer, W. Critical inquiry in a text-based environment: Computer conferencing in higher education. The Internet and Higher Education 2(2-3): 87-105, 1999.)

Cognitive presence concerns the construction of meaning and confirmation of understanding. Social presence encompasses the ability of participants to coalesce for a common purpose. Considering the asynchronous virtual community in which students interact, this may demand a significant role adjustment. Teaching presence must manage and monitor the cognitive and social dynamic to create a purposeful community of inquiry. This requires recognizing the unique features of the medium, capitalizing on them to achieve intended educational experiences, and attending to the inevitable role identity adjustment of the students. Finally, it is validating and revealing to note the three overlapping areas of each pair of elements. They represent three key responsibilities and features of an authentic educational experience.

Key indicators for each of the elements were generated and tested to explicate the elements and provide a means to study the nature of online learning experiences. The indices can be used to operationalize the community of inquiry model in terms of assessing online transcripts and development more objective instruments. They could also be used to diagnose an educational transaction to optimize teaching interventions. For a full listing of indicators see Garrison and Anderson [1].

Role identity adjustment cannot be ignored. Inevitably, the student must assume greater responsibility to match the increased control that comes with online learning. This is compounded by the intellectual demands of the precision of written communication. In combining both the freedom and demands of online communication, participants must move from a relatively passive classroom experience into a more active online community of inquiry. 


\section{B. Role Adjustment}

The role assumed by the online learner is one of both independence and interdependence. This role and its associated responsibilities represent higher standards that more closely match those of life outside the classroom. Educationally, this is a considerable advantage as students must become more self-directed and learn to learn. However, these challenges and role adjustments need to be understood and managed if students are to be successful in an online community of inquiry, and beyond. Students must assume a new role identity and this may not be an easy adjustment [6].

Role is used here as a sociological construct, defined as a collection of behavioral requirements associated with a certain social position in a group, organization or society [7]. At its most general level, role expectations are dictated by the social structure. Individuals who engage in the role are guided, through a process of socialization, to appropriate role performance. Socialization then refers to the "process by which people learn the characteristics of their group ... (and) the attitudes, values and actions thought appropriate for them" [8].

Under conditions of long-standing roles, individuals engage in 'role-taking' behavior, where observation and mimicry of role models allow those new to the role to 'practice' appropriate role behaviors. 'Role making' occurs as individuals construct aspects of the role with their own individual meanings and satisfying behaviors attached. This occurs under social conditions where such individual autonomy is allowed. It also occurs where role models are not readily available, and construction of the role is required.

Such is the case for becoming an online learner. An adjustment from the more generalized role of learner, the responsibilities and requirements of working online are not readily apparent to those new to the role. The transition to, and adjustment in, the role of online learner, is part of the current social climate in online learning. While maintaining the usual expectations and privileges attached to the role of learner, online learners add such things as:

- knowledge about, skill with, and acceptance of the technology

- new modes and amounts of communication with instructors, peers and administrators

- increased levels of learner self-direction, and

- a new 'place' for learning in time (anytime, usually determined by the learner and their life circumstances) and space (anywhere, dependent upon equipment requirements).

Role is used here to define certain behavioral competencies required to be a functioning member of a community [9]. Normalizing a new role requires the development of the appropriate competencies characteristic of the structure, values and actions of the community. This socialization process occurs through guidance from the community and provides the opportunity for adjustment.

Moving into the role of online learner will necessitate a "role making" process where the individual must assume considerable responsibility to construct a role with personal meaning. This is consistent with the asynchronous nature of the online community and the control and freedom characteristic of the medium. This more active and participatory role making process associated with becoming an online learner is not readily apparent nor its meaning easily constructed.

The adjustment to online learner goes well beyond the technical skill adjustment. Online students must 
learn to communicate and become familiar with other members of the community through a medium without the visual cues afforded in a face-to-face setting. The cognitive demands may well also increase as learners are expected to contribute ideas and share their thoughts, which are made permanent in the process. As this occurs, social identity will begin to change. Identifying with the role of online learner will inevitably require adjustment.

Role identity adjustment is acquired in, and facilitated by, the online community. Although it may be difficult to discern what 'community' really means to online learners [10], the development of online community is a necessity for the role identity acquisition of online learners. While all members of the community participate in this role adjustment, deliberate action is required in terms of teaching presence. Students will need to be made explicitly aware of certain role requirements. The student must reflectively accommodate other subtler role adjustments. This iterative comparison of one's own behavior to others is core to role identity adjustment.

\section{METHODOLOGY}

The instrument was constructed from the empirically confirmed indicators of each of the three elements of the community of inquiry model [1]. Items were written to correspond to the indices. Items 1-8 are intended to reflect cognitive presence; items 9-15 are intended to reflect social presence; and items 16-28 are intended to reflect teaching presence. For a more detailed discussion of the indices and examples see Garrison and Anderson [1].

This study developed and tested an instrument to measure the extent of their identification with the behaviors, expectations and requirements of the role of online learner. Two forms of the questionnaire containing 28 Likert-type questions examining role identity were constructed (see Appendix A). The first form of the questionnaire measured the students' anticipated personal adjustment to online learning compared to their previous face-to-face learning experiences. The second form measured the students' anticipated personal adjustment to online learning compared to their perceived experienced online learners. Five response choices were provided ranging from much better to much worse.

The study was conducted in the winter term of 2003 with participants in two graduate programs at Athabasca University. The goal was to examine the expectations of learners prior to experiencing online learning. While experience was limited, it was expected that learners had a 'straw-model,' or rough idea, of online learning activities as a point of reference. Respondents for the main study were drawn from courses purposively sampled according to position in a program of studies. As this research tests the adoption of role identity adjustment as students make the transition to online learning, courses early in the students program of studies were used to collect data. Students from six distinct courses were included. The total number of students included was 65 .

All courses were delivered with a combination of print and electronic material and online conferencing. It is important to note that online conferencing constituted the central mechanism for student engagement and group interaction. Students in these courses were unknown to one another in all but a few cases. Students were not part of a cohort, nor do they participate in courses with the same group of students over time. Online conferencing (i.e., discussion groups) occurred in different frequencies across courses. Two sections had weekly conferences, while four sections had five weekly conferences across thirteen weeks. Conferences were instructor led and mandated in all sections.

Collegial review of the instrument indicated high face validity. An initial sample of 25 students was 
drawn to pilot the instrument. This group was representative of the population but did not include respondents in the research sample. Pilot respondents provided feedback on syntax, word usage, and comprehensive coverage of activities. Reliability for single test administration was evaluated using Cronbach's Alpha. Results indicated high reliability for the experienced online learner comparison and acceptable reliability for the face-to-face comparison (see Table 1 below).

Table 1: Pilot Reliability Data

\begin{tabular}{|l|c|c|}
\hline INSTRUMENT & Alpha & Number of Items \\
\hline Experienced Online Learners & 0.9681 & 28 \\
\hline Face-to-Face & 0.6393 & 28 \\
\hline
\end{tabular}

Each consenting student was sent the two forms of the questionnaire by e-mail at the beginning of the course. The first questionnaire asked students to assess the anticipated quality of his/her participation in multiple activities in the online community as compared to the quality of participation previously experienced in face-to-face learning environments. Respondents indicated a response by choosing one of five choices on a continuum from 'much better' to 'much worse.' The second questionnaire presented the same set of learning activities, but students were asked to assess the anticipated quality of his/her participation in multiple activities in the online community as compared to the perceived activities of experienced online learners. Role identity adjustment occurs through the process of social referents; this process of data collection and analysis allows us to compare identity adjustment from two likely central referents in the development of online learner role identity.

Preliminary analyses by way of frequencies distribution and descriptive statistics for all variables were performed. Factor analysis was then employed to explore construct validity. Reliability analysis was performed to determine the internal consistency of each factor.

\section{RESULTS}

The purpose of the exploratory factor analysis was to assess the underlying structure of the community of inquiry instrument used to measure role identity adjustment prior to an online learning experience. Students completed the questionnaire by comparing their anticipated adjustment from two perspectivesprevious face-to-face learning experiences and perceived experienced online learners.

Eigenvalues were generated by a factor extracting procedure. There were five factors with eigenvalues greater than one. To explore the underlying structure of the variables for the face-to-face (F2F) and experienced online learner (EOL) questionnaires, both orthogonal and non-orthogonal rotation of the five and four factor solutions were obtained. Neither provided the interpretability or simple structure desired. Therefore, a three-factor oblimin solution was chosen. This provided simple structure and, most importantly, corresponded best to the theoretical model. The oblimin solution was also expected to be the better solution as the three factors of the community of inquiry are seen to be overlapping. That is, they do not exist in isolation from the other elements in a proper educational experience. Salient loadings (>.30) of the three factor solutions for both versions of the questionnaire can be found in Tables 1 and 2. 
Table 2: Compared to Experienced Online Learners-Sorted Rotated (oblimin) Factor Loadings from Principal Components

FACTOR LOADINGS

\section{Factor 1: Cognitive Presence?}

V05:Synthesize ideas

V08:Apply ideas or concepts

V02:Stimulate your curiosity

V07:Confirm concept understanding

V17:Know how to participate

V03: Identify relevant new

Information

V01:Understand the issues being

Presented

V16:Understand expectations

V18:Take responsibility

Factor 2: Social Presence?

V09:Express your emotions

V10:Be open \& disclose personality

V12:Respond to others' comments

V13: Sustain discussion

V14:Feel part of the class

Community

V21:Feel comfortable engaging

In discussion

V11:Ask questions

V04:Engage in exchange of ideas

V06:Generate tentative solutions

V15:Refer to others by name

Factor 3: Teaching Presence?

V27:Accepting teacher assessment

V28:Accepting teacher feedback

V25:Teaching assistance in Reaching consensus

V26:Direct teaching intervention

V24:Teacher interaction

V23:The organization of the class .35460

V20:Adjust to the climate .42810

V22: Teaching methods

V19:Adjust to the context

Eigenvalue

Variance explained

\section{Factor 1}

.88812

.84051

.68938

.68067

.66219

.66205

.63784

.59935

.50501
Factor 2 Factor 3

.91686

.83194

.74985

.71960

.66707

.59259

.57835

.53943

.50743

.50448

.40252

.93215

.91252

.81487

.77659

.63121

.61882

.53860

$.36189 \quad .51487$

.50206

$2.60181 \quad 1.91182$

$9.3 \quad 6.8$ 
Table 3: Compared to Face to Face-Sorted Rotated (oblimin) Factor Loadings from Principal Components

FACTOR LOADINGS

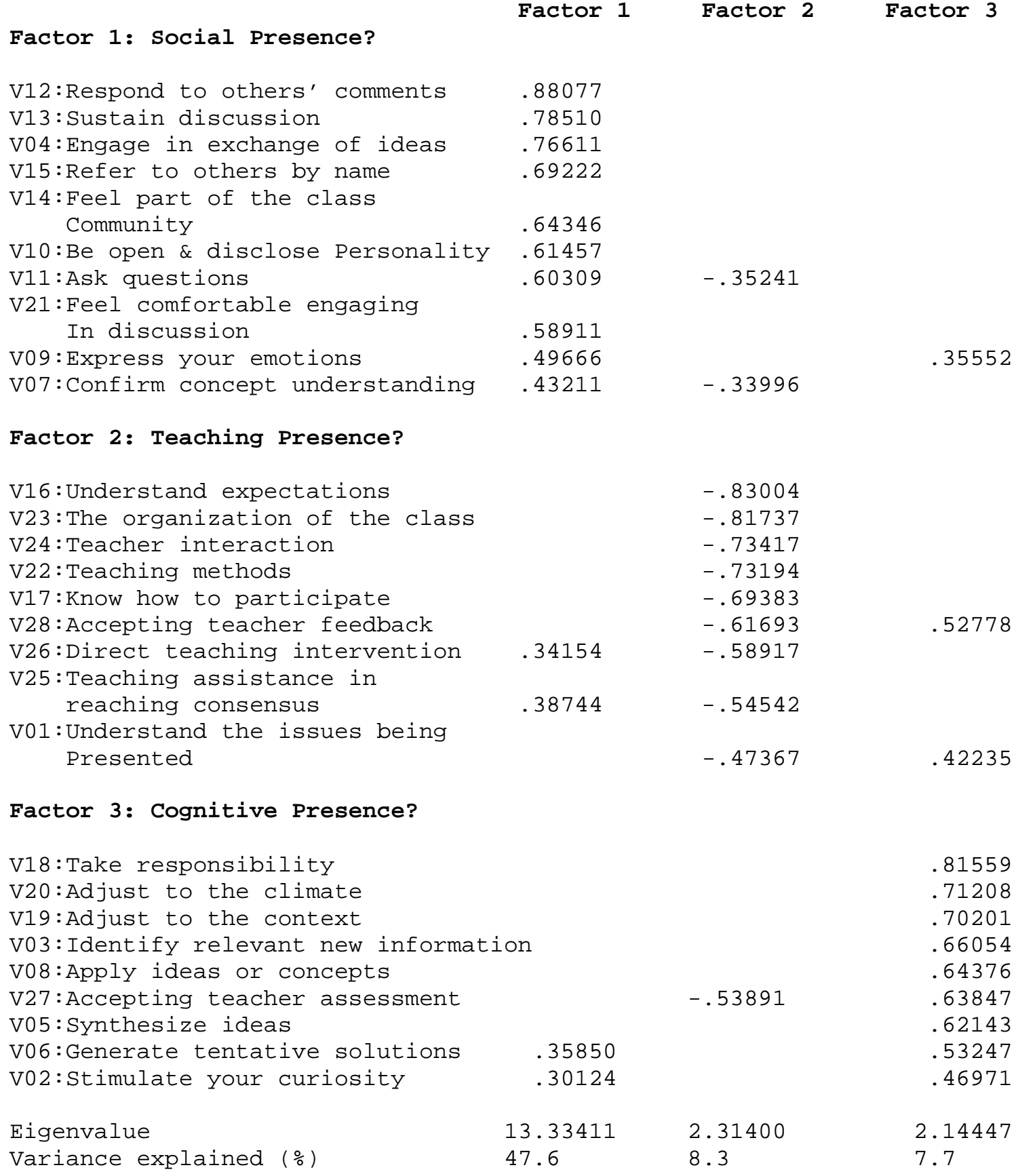

\section{A. Experienced online learners}

The factor structure for the experienced online learner solution corresponded well to the hypothesized model. Although not all items loaded as predicted, the solution clearly corresponds to the theoretical constructs of cognitive, social and teaching presence respectively. The first factor should be labeled 
cognitive presence. Three variables were not congruent with the projected loadings. These variables were: understand expectations (v16), know how to participate (v17) and, take responsibility (v18). They were expected to load on teaching presence. First, it should be noted that they did not load saliently on other factors. Secondly, upon reflection, "understand expectations" and "take responsibility" were seen as best reflecting cognitive presence. The variable, "know how to participate" could be interpreted as reflecting social presence. We suggest that this was not the case as entering students expected participation to mean becoming involved in the learning experience.

The second factor was labeled social presence. All but three variables loaded as expected. The three variables not projected to load on this factor were; engage in exchange of ideas (v4), generate tentative solutions (v6), and, feel comfortable engaging in discussion (v21). The question is whether they can be reasonably interpreted as being congruent with social presence. Certainly, these three items are not inconsistent with social presence. Each of these variables would appear to have both social and cognitive presence in that they involve engagement with the group for learning purposes. The multiple loadings of variables four and six reflect the dual social and cognitive nature of these items and the inherent overlap of the elements (i.e., factors) of the theoretical model.

The third factor was labeled teaching presence. All the variables that did load on this factor were projected to load here. At the same time, as was noted previously, variables 16, 17, 18, and 21 had been projected to load on the teaching presence factor but loaded elsewhere. It is suggested that this was due to ambiguous wording.

\section{B. Face-to-face learning experiences}

The role adjustment questionnaire comparing to previous face-to-face learning experiences resulted in a factor structure similar to the EOL factor structure. The primary exception to this is the order of the factors. The first factor was identified as social presence and had similar variable loadings with the exception that variable six and seven switched between social and cognitive presence compared to the EOL factor structure. The second factor was clearly interpreted as teaching presence as it loaded with all hypothesized teaching presence variables with the exception of the first variable- "understanding the issues presented". This variable had a multiple loading with the third factor (cognitive presence) where it was projected to load.

The third factor, as alluded to previously, is labeled cognitive presence. While this factor does have five factors hypothesized to load on cognitive presence, there are several variable loadings that give some difficulty in interpreting this factor. The first three (i.e., highest loading) variables, in addition to the sixth loading variable, were hypothesized to load on teaching presence. The first of these, taking responsibility, does not create a challenge in terms of interpreting it as cognitive presence. This variable loaded on cognitive presence in the EOL factor structure and its interpretation was justified previously. Variable 27, accepting teacher assessment, had a salient loading on both cognitive and teaching presence. It was hypothesized to load on teaching presence so this is not considered a serious challenge to the interpretation of this factor as cognitive presence.

However, variables 19 and 20 are difficult to explain as to why they loaded here. We believe the root of cause is in the poor phrasing of the items. These items were not phrased in a way that was consistent with the particular indices that they were based upon. For example, the original teaching presence indicee that these were based upon was "setting climate for learning" [1, p. 70]. 
Based upon the factor loadings, a revised instrument was constructed (see Appendix B). The items retained were the top loading items that were common to each of the factor loadings across the two versions of the questionnaire. Suggested revised items (in italics) replaced those that were not common to loadings for each factor. As in the original questionnaire, items 1-8 are intended to reflect cognitive presence; items 9-15 are intended to reflect social presence; and, items 16-28 are intended to reflect teaching presence.

Reliability of the factors (Cronbach's alpha) was high and acceptable (see Table 3).

Table 4: Reliability coefficients (Cronbach's alpha) of each factor

\begin{tabular}{|l|c|c|c|c|c|c|}
\hline \multirow{2}{*}{ AREAS } & \multicolumn{5}{|c|}{ FACTORS } \\
\cline { 2 - 7 } & Social Presence & \multicolumn{2}{c|}{ Teaching Presence } & \multicolumn{2}{c|}{ Cognitive Presence } \\
\cline { 2 - 7 } & Alpha & $\begin{array}{c}\text { No. of } \\
\text { items }\end{array}$ & Alpha & $\begin{array}{c}\text { No. of } \\
\text { items }\end{array}$ & Alpha & $\begin{array}{c}\text { No. of } \\
\text { items }\end{array}$ \\
\hline $\begin{array}{l}\text { Experienced } \\
\text { online learners }\end{array}$ & 0.9237 & 10 & 0.9452 & 9 & 0.9389 & 9 \\
\hline Face-to-Face & 0.9211 & 10 & 0.9241 & 9 & 0.9070 & 9 \\
\hline
\end{tabular}

\section{DISCUSSION}

The purpose of the study reported here was to validate the instrumentation to assess role adjustment for online learning. The study assessed students' anticipated personal adjustment to online learning by comparing to both previous face-to-face learning experiences and to perceived experienced online learners. While both points of comparison revealed the same educational factor structure (i.e., cognitive, social and teaching presence), the order of the factors and variance accounted for were different. That is, the face-to-face comparison focused first on social and teaching presence, which explained 47.6 and 8.3 percent of the variance. On the other hand, when comparing to the experienced online learner, students focused first on cognitive presence, which explained 52.7 percent of the variance.

The factor order would suggest that students do see a difference in the learning process and a need for role adjustment. Moreover, it would suggest that a face-to-face learning experience is viewed as more externally oriented (i.e., social and teaching presence), while online learning is viewed as more cognitive or internally oriented. Thus, online learning would be perceived as requiring greater individual responsibility. An interesting question is whether cognitive presence is perceived as less of a concern in face-to-face compared to online learning. That is, traditional classrooms may be seen largely as a place of information transmitted by a teacher to a class. Assimilation of the content is, therefore, a separate and individual process separate from the face-to-face classroom experience. This would be in contrast to online learning which is concurrent with and integral to the learning process. Therefore, online learning might be perceived as requiring increased responsibility for one's learning.

If this explanation has validity, it would have implications for the quality of learning outcomes (i.e., deep and surface approaches). Online learning may be perceived as congruent with deep approaches to learning and higher quality learning outcomes. If this were the case, role adjustment would be a significant challenge for students engaging in online learning. Regardless, the initial findings of this study suggest that students do perceive face-to-face and online learning differently. The challenge for future research is to understand how engagement in an online learning community affects the perception of role identity. 
Also, how does perceived online learning roles influence the approaches to learning and the quality of learning outcomes.

Finally, the instrument provides a means to assess and study the magnitude of adjustment during the progression of an online course from the perspective of cognitive, social and teaching presence. Initial findings of one such study have been reported [11]. This type of study could have enormous theoretical and practical benefit in terms of understanding and facilitating the cognitive, social and teaching support students need as they adjust to an online learning environment. Much work will be required to refine our understanding of design and support in a variety of disciplinary and institutional contexts. Rigorous and systematic research into online learning is predicated upon the availability of validated instruments.

\section{REFERENCES}

1. Garrison, D. R. and T. Anderson. E-Learning in the $21^{\text {st }}$ Century: A Framework for Research and Practice. London: Routledge/Falmer, 2003.

2. Brown, J. S. Growing Up Digital: How the Web Changes Work, Education, and the Ways People Learn. Change 32(2): 11-20, March/April 2000.

3. Garrison, D. R., T. Anderson and W. Archer. Critical Inquiry in a Text-Based Environment: Computer Conferencing in Higher Education. The Internet and Higher Education 2(2-3): 87-105, 2000.

4. Collier, P. A Differentiated Model of Role Identity Acquisition. Symbolic Interactionist 24(2): 217235, 2001.

5. Garrison, D. R., T. Anderson and W. Archer. Critical Thinking, Cognitive Presence and Computer Conferencing in Distance Education. American Journal of Distance Education 15(1): 7-23, 2001.

6. Cleveland-Innes, M., and D. R. Garrison. Becoming a Member of an Online Community: Role Identity Acquisition for Online Learners. Unpublished manuscript.

7. Kendall, D., J. Murray and R. Linden. Sociology in Our Times (2nd ed.). Ontario: Canadian Cataloguing in Publication, 2000.

8. Kanwar, M. and D. Swenson. Canadian Sociology. Iowa: Kendall/Hunt Publishing Company, 2000.

9. Blau, J. R. and N. Goodman (eds.). Social Roles and Social Institutions. New Brunswick: Transaction Publishers, 1995.

10. Conrad, D. Deep in the Hearts of Learners: Insights into the Nature of Online Community. Journal of Distance Education 17(1): 1-19, 2002.

11. Garrison, D. R., and M. Cleveland-Innes. Critical Factors in Student Satisfaction and Success: Facilitating Student Role Adjustment in Online Communities of Inquiry. In Elements of Quality Online Education: Into the Mainstream, Vol 4 in the Sloan-C Series, eds J. Bourne and J. Moore, 2938. Needham, MA: Sloan-C, forthcoming, 2004.

\section{ABOUT THE AUTHORS}

Dr. D. Randy Garrison is the Director of the Learning Commons at the University of Calgary. He is also a full professor in the Faculty of Education. He served as Dean, Faculty of Extension at the University of Alberta from 1996 to 2001. Randy has published extensively on teaching and learning in distance, higher, and adult education contexts.

Dr. Martha Cleveland-Innes has served as a practitioner, researcher and facilitator of learning for more than two decades in the field of adult, higher and continuing education. She is currently a faculty member in the Centre for Distance Education at Athabasca University. Her research interests are social factors in student approaches to learning, student role adjustment in online environments, affective learning 
outcomes in distance education and learning in the workplace.

\section{APPENDIX A - ORIGINAL QUESTIONNAIRE}

This instrument is designed to assess your experiences in online learning. The following questions will assist us in assessing your perceptions with regard to learning in an online environment. Your responses will be held in strict confidence and your identity will not be revealed to anyone other than the researchers in the project. Please complete all pages of this questionnaire. This will take approximately 20 minutes.

Section A.: Please answer the following questions by placing an ' $\mathrm{X}$ ' in the appropriate response box.

Compared to previous face-to-face learning experiences, how would you rate your online learning experiences with the following?

\begin{tabular}{|c|c|c|c|c|c|}
\hline \multirow[t]{2}{*}{ ACTIVITY } & \multicolumn{5}{|c|}{ RESPONSE } \\
\hline & $\begin{array}{l}\text { Much } \\
\text { Better }\end{array}$ & Better & Same & Worse & $\begin{array}{l}\text { Much } \\
\text { Worse }\end{array}$ \\
\hline \multicolumn{6}{|c|}{$\begin{array}{l}\text { Understanding the issues and problems } \\
\text { being presented? }\end{array}$} \\
\hline \multicolumn{6}{|l|}{ Stimulating your curiosity? } \\
\hline \multicolumn{6}{|c|}{ Identifying relevant new information? } \\
\hline \multicolumn{6}{|l|}{ Engaging in exchange of ideas? } \\
\hline \multicolumn{6}{|l|}{ Synthesizing ideas? } \\
\hline \multicolumn{6}{|c|}{$\begin{array}{l}\text { Generating tentative solutions or resolution } \\
\text { to problems? }\end{array}$} \\
\hline \multicolumn{6}{|c|}{ Confirming understanding of concepts? } \\
\hline \multicolumn{6}{|l|}{ Applying ideas or concepts? } \\
\hline \multicolumn{6}{|l|}{ Expressing your emotions? } \\
\hline \multicolumn{6}{|c|}{$\begin{array}{l}\text { Being open? } \\
\text { (i.e. disclosing your personality) }\end{array}$} \\
\hline \multicolumn{6}{|l|}{ Asking questions? } \\
\hline \multicolumn{6}{|c|}{ Responding to others' comments? } \\
\hline \multicolumn{6}{|l|}{ Sustaining discussion? } \\
\hline \multicolumn{6}{|c|}{ Feeling part of the class community? } \\
\hline \multicolumn{6}{|l|}{ Referring to others by name? } \\
\hline \multicolumn{6}{|l|}{ Understanding expectations? } \\
\hline Knowing how to participate? & & & & & \\
\hline
\end{tabular}




\begin{tabular}{|l|}
\hline Taking responsibility? \\
\hline Adjusting to the online context? \\
\hline Adjusting to the learning climate? \\
\hline Feeling comfortable engaging in discussion? \\
\hline Feeling comfortable with teaching methods? \\
\hline $\begin{array}{l}\text { Enderstanding organization of the class? } \\
\text { (questions, comments, facilitation)? }\end{array}$ \\
\hline $\begin{array}{l}\text { Receiving teacher assistance in reaching } \\
\text { consensus? }\end{array}$ \\
\hline Receiving teaching intervention? \\
\hline Accepting teacher assessment?
\end{tabular}

Section B: Please answer the following questions by placing an ' $\mathrm{X}$ ' in the appropriate response box.

Compared to experienced online learners, how would you rate your online learning experiences with the following?

(QUESTIONNAIRE REPEATED)

\section{APPENDIX B - REVISED QUESTIONNAIRE}

This instrument is designed to assess your experiences in online learning. The following questions will assist us in assessing your perceptions with regard to learning in an online environment. Your responses will be held in strict confidence and your identity will not be revealed to anyone other than the researchers in the project. Please complete all pages of this questionnaire. This will take approximately 20 minutes.

Section A.: Please answer the following questions by placing an ' $\mathrm{X}$ ' in the appropriate response box.

Compared to previous face-to-face learning experiences, how would you rate your online learning experiences with the following?

\begin{tabular}{|l|l|l|l|l|l|}
\hline \multicolumn{1}{|c|}{ ACTIVITY } & \multicolumn{3}{c|}{ RESPONSE } \\
\hline & $\begin{array}{l}\text { Much } \\
\text { Better }\end{array}$ & Better & Same & Worse & $\begin{array}{c}\text { Much } \\
\text { Worse }\end{array}$ \\
\hline & & & & & \\
\hline Identify key issues & & & & & \\
\hline Stimulating your curiosity? & & & & & \\
\hline
\end{tabular}




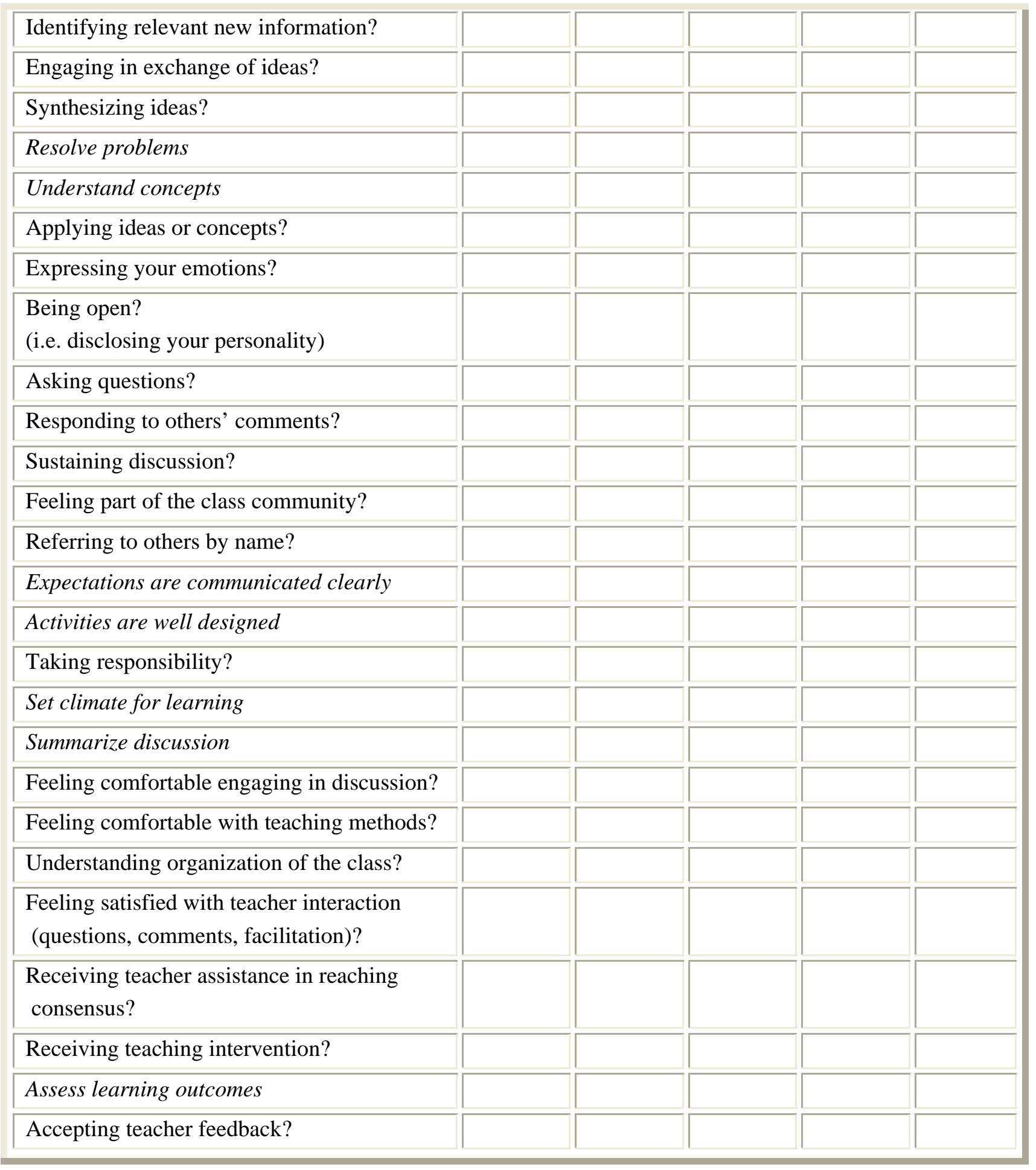

Section B: Please answer the following questions by placing an ' $\mathrm{X}$ ' in the appropriate response box.

Compared to experienced online learners, how would you rate your online learning experiences with the following? 